\title{
Aromaticity-Driven Rupture of CN Triple and CC Double Bonds: Mechanism of the Reaction Between $\mathrm{Cp}_{2} \mathrm{Ti}\left(\mathrm{C}_{4} \mathrm{H}_{4}\right)$ and $\mathrm{RCN}$
}

\author{
Cherumuttathu $\mathrm{H}$. Suresh ${ }^{\dagger, *}$ and Nobuaki Koga ${ }^{\ddagger}$, \\ ${ }^{\dagger}$ Computational Modeling and Simulation Section, Regional Research Laboratory (CSIR), \\ Thiruvananthapuram 695 019, India \\ ${ }^{*}$ Graduate School of Information Science, Nagoya University, Nagoya 464-8601, Japan
}

\section{Supporting Information}

Table 1. Energies in a.u. at B3LYP/Gen1 level.

$\begin{array}{cllll}\text { Molecule } & \begin{array}{l}\text { Sum of electronic } \\ \text { and zero-point }\end{array} & \begin{array}{l}\text { Sum of electronic } \\ \text { and thermal }\end{array} & \begin{array}{l}\text { Sum of electronic } \\ \text { and thermal }\end{array} & \begin{array}{l}\text { Sum of electronic } \\ \text { and thermal }\end{array} \\ & \text { Energies } & \text { Energies } & \text { Enthalpies } & \text { Free Energies } \\ \text { Benzene } & -232.143024 & -232.138638 & -232.137694 & -232.170485 \\ \text { Acetonitrile } & -132.705839 & -132.702246 & -132.701302 & -132.729854 \\ \text { 1 } & -599.718503 & -599.704836 & -599.703891 & -599.758795 \\ \text { TS1 } & -599.681554 & -599.669146 & -599.668202 & -599.719494 \\ \mathbf{2} & -599.687997 & -599.674784 & -599.673840 & -599.728145 \\ \text { TS2 } & -599.680820 & -599.668476 & -599.667532 & -599.719878 \\ \mathbf{3} & -599.693125 & -599.680449 & -599.679505 & -599.732358 \\ \text { TS3 } & -599.681109 & -599.669014 & -599.668070 & -599.719116 \\ \mathbf{4} & -599.688872 & -599.675847 & -599.674903 & -599.728206 \\ \text { TS4 } & -599.682158 & -599.668811 & -599.667867 & -599.722793 \\ \mathbf{5} & -599.700928 & -599.686101 & -599.685157 & -599.745107 \\ \mathbf{6} & -500.290414 & -500.276846 & -500.275901 & -500.333365 \\ \text { TS5 } & -500.279469 & -500.266646 & -500.265702 & -500.319613 \\ \mathbf{7} & -500.333120 & -500.321363 & -500.320419 & -500.372116 \\ \mathbf{8} & -633.064434 & -633.048262 & -633.047318 & -633.108322 \\ \text { TS6 } & -633.064321 & -633.048972 & -633.048028 & -633.106913 \\ \mathbf{9} & -633.081498 & -633.066267 & -633.065323 & -633.125596\end{array}$




$\begin{array}{ccccc}\text { TS7 } & -633.045537 & -633.031311 & -633.030366 & -633.087500 \\ \mathbf{1 0} & -633.047055 & -633.032485 & -633.031541 & -633.089433 \\ \text { TS8 } & -633.032622 & -633.018123 & -633.017179 & -633.074824 \\ \mathbf{1 1} & -633.062865 & -633.047126 & -633.046182 & -633.107859 \\ \mathbf{1 2} & -765.794329 & -765.773662 & -765.772718 & -765.846818 \\ \text { TS9 } & -765.774848 & -765.756096 & -765.755152 & -765.821644 \\ \mathbf{1 3} & -765.788959 & -765.770399 & -765.769454 & -765.835147 \\ \text { TS10 } & -765.784374 & -765.766929 & -765.765985 & -765.829335 \\ \mathbf{1 4} & -765.791563 & -765.773759 & -765.772815 & -765.837023 \\ \text { TS11 } & -765.776190 & -765.758513 & -765.757569 & -765.821044 \\ \mathbf{1 5} & -765.836487 & -765.818451 & -765.817507 & -765.882747\end{array}$

Optimized geometries at B3LYP/Gen1 level (Atomic number and the $\mathrm{X}, \mathrm{Y}, \mathrm{Z}$ Cartesian coordinates)

$\begin{array}{lccc}\text { 1. } & & & \\ 22 & -0.000682 & -0.082143 & -0.002984 \\ 6 & 0.0864260 & 1.523932 & 1.3775920 \\ 6 & -0.070259 & 1.5288650 & -1.378092 \\ 6 & -0.028341 & 2.7168850 & -0.730146 \\ 6 & 0.0564070 & 2.714307 & 0.7334810 \\ 6 & -2.179362 & -0.079622 & 0.9535670 \\ 6 & -2.348096 & 0.1917770 & -0.429162 \\ 6 & -1.959866 & -0.966347 & -1.1551700 \\ 6 & -1.547831 & -1.946328 & -0.2274650 \\ 6 & -1.666536 & -1.399345 & 1.0782150 \\ 6 & 2.1832760 & -0.094927 & -0.945695 \\ 6 & 2.3477800 & 0.165081 & 0.4395190 \\ 6 & 1.9432910 & -0.993763 & 1.155575 \\ 6 & 1.6574630 & -1.408495 & -1.082108 \\ 6 & 1.5264040 & -1.963113 & 0.218995 \\ 1 & 0.1818960 & 1.489160 & 2.4659860 \\ 1 & -0.165047 & 1.4991910 & -2.466737 \\ 1 & -0.071769 & 3.6761790 & -1.254964 \\ 1 & 0.1099870 & 3.671426 & 1.2612890 \\ 1 & -2.407651 & 0.5969650 & 1.763419 \\ 1 & -2.704790 & 1.1174130 & -0.854646 \\ 1 & -1.943379 & -1.062195 & -2.2336740 \\ 1 & -1.179044 & -2.933635 & -0.4721280 \\ 1 & -1.416779 & -1.902766 & 2.0038530 \\ 1 & 2.4213520 & 0.585085 & -1.7498120\end{array}$




$\begin{array}{lllr}1 & 2.7117780 & 1.0842470 & 0.872737 \\ 1 & 1.920703 & -1.096364 & 2.233328 \\ 1 & 1.406678 & -1.902652 & -2.012481 \\ 1 & 1.146529 & -2.948351 & 0.454849\end{array}$

\section{TS1}

$\begin{array}{llll}22 & 0.243934 & 0.118445 & -0.205426\end{array}$

$\begin{array}{lllll}6 & -1.746078 & -0.515918 & 1.24868\end{array}$

$\begin{array}{llll}6 & -0.784313 & -1.396241 & -1.25942\end{array}$

$\begin{array}{llll}6 & -1.777945 & -2.099568 & -0.63489\end{array}$

$\begin{array}{llll}6 & -2.079681 & -1.739277 & 0.71827\end{array}$

$\begin{array}{llll}6 & -1.828735 & 0.921218 & 0.587948\end{array}$

$\begin{array}{llll}6 & -1.650551 & 1.038760 & -0.916517\end{array}$

$\begin{array}{llll}6 & -0.680769 & 2.037469 & -1.208887\end{array}$

$\begin{array}{lllll}6 & -0.076502 & 2.463956 & -0.013033\end{array}$

$\begin{array}{llll}6 & -0.640314 & 1.741920 & 1.060487\end{array}$

$\begin{array}{llll}1 & -1.831658 & -0.403776 & 2.32999\end{array}$

$\begin{array}{lllll}1 & -0.541557 & -1.694991 & -2.28887\end{array}$

$\begin{array}{lllll}1 & -2.290626 & -2.953311 & -1.08418\end{array}$

$\begin{array}{llll}1 & -2.504214 & -2.496157 & 1.38371\end{array}$

$\begin{array}{llll}1 & -2.816677 & 1.285914 & 0.926268\end{array}$

$\begin{array}{llll}1 & -2.353835 & 0.635027 & -1.628421\end{array}$

$\begin{array}{llll}1 & -0.401340 & 2.365851 & -2.204076\end{array}$

$\begin{array}{llll}1 & 0.745861 & 3.165614 & 0.056004\end{array}$

$\begin{array}{llll}1 & -0.399279 & 1.880771 & 2.107465\end{array}$

$\begin{array}{llll}6 & 1.826523 & -1.671595 & -0.110296\end{array}$

$\begin{array}{llll}6 & 1.734335 & -1.128838 & 1.193458\end{array}$

$\begin{array}{llll}6 & 2.197571 & 0.217278 & 1.142744\end{array}$

$\begin{array}{llll}6 & 2.328715 & -0.655629 & -0.969636\end{array}$

$\begin{array}{llll}6 & 2.576306 & 0.509406 & -0.194464\end{array}$

$\begin{array}{lllll}1 & 1.542018 & -2.671981 & -0.403303\end{array}$

$\begin{array}{lllll}1 & 1.363711 & -1.643506 & 2.070152\end{array}$

$\begin{array}{llll}1 & 2.250566 & 0.900100 & 1.980591\end{array}$

$\begin{array}{llll}1 & 2.487676 & -0.754096 & -2.037474\end{array}$

$\begin{array}{llll}1 & 2.991415 & 1.441105 & -0.555127\end{array}$

2.

$\begin{array}{llll}22 & -0.433297 & -0.068322 & -0.248510\end{array}$

$\begin{array}{llll}6 & 2.754328 & 0.173972 & 0.9633650\end{array}$

$\begin{array}{llll}6 & 0.749995 & 1.486213 & -1.0050110\end{array}$

$\begin{array}{llll}6 & 1.877987 & 2.041543 & -0.4932220\end{array}$

$\begin{array}{llll}6 & 2.710723 & 1.460279 & 0.5519140\end{array}$

$\begin{array}{llll}6 & 2.006413 & -1.005074 & 0.414666\end{array}$

$\begin{array}{llll}6 & 0.625801 & -1.353660 & 1.053139\end{array}$

$\begin{array}{llll}6 & -0.048663 & -2.209147 & 0.086922\end{array}$

$\begin{array}{llll}6 & 0.558313 & -1.992414 & -1.180198\end{array}$ 


$\begin{array}{rrrr}6 & 1.588374 & -1.056997 & -1.064677 \\ 1 & 3.429438 & -0.070713 & 1.781784 \\ 1 & 0.293577 & 2.053789 & -1.8287830 \\ 1 & 2.217475 & 3.016599 & -0.8555840 \\ 1 & 3.394336 & 2.151896 & 1.0463670 \\ 1 & 2.674335 & -1.878496 & 0.563201 \\ 1 & 0.529115 & -1.495165 & 2.124780 \\ 1 & -0.848243 & -2.910546 & 0.287252 \\ 1 & 0.178790 & -2.380239 & -2.122724 \\ 1 & 2.215736 & -0.715832 & -1.876113 \\ 6 & -1.995132 & 1.672649 & 0.073364 \\ 6 & -2.522809 & 0.749053 & -0.873356 \\ 6 & -2.795860 & -0.475437 & -0.196617 \\ 6 & -1.919675 & 1.005625 & 1.322834 \\ 6 & -2.418930 & -0.310376 & 1.158463 \\ 1 & -1.687981 & 2.688993 & -0.126116 \\ 1 & -2.700327 & 0.949440 & -1.923868 \\ 1 & -3.212728 & -1.371387 & -0.639083 \\ 1 & -1.517754 & 1.419081 & 2.239914 \\ 1 & -2.458419 & -1.071699 & 1.926674\end{array}$

$\begin{array}{lrcr}\text { TS2 } & & & \\ 22 & -0.527442 & -0.1672370 & -0.268698 \\ 6 & 2.798807 & 0.291599 & 1.0096240 \\ 6 & 0.903366 & 1.087946 & -1.1012860 \\ 6 & 1.842177 & 1.930549 & -0.5665860 \\ 6 & 2.681081 & 1.559658 & 0.5452240 \\ 6 & 2.077598 & -0.887700 & 0.4512490 \\ 6 & 0.698595 & -1.277664 & 1.0876170 \\ 6 & 0.084051 & -2.190976 & 0.1444130 \\ 6 & 0.617786 & -1.877753 & -1.1421780 \\ 6 & 1.601242 & -0.827766 & -1.0210700 \\ 1 & 3.475907 & 0.089785 & 1.8371560 \\ 1 & 0.500854 & 1.347791 & -2.0962160 \\ 1 & 2.023182 & 2.903136 & -1.0267950 \\ 1 & 3.295230 & 2.339817 & 0.9926930 \\ 1 & 2.749841 & -1.761851 & 0.5360240 \\ 1 & 0.602379 & -1.406967 & 2.1613690 \\ 1 & -0.611965 & -2.9932520 & 0.356497 \\ 1 & 0.305569 & -2.312298 & -2.0874810 \\ 1 & 2.296378 & -0.647604 & -1.8348500 \\ 6 & -2.233862 & 1.514687 & -0.4151220 \\ 6 & -2.771812 & 0.247417 & -0.7931590 \\ 6 & -2.765711 & -0.5952850 & 0.352904 \\ 6 & -1.881873 & 1.444881 & 0.9497740 \\ 6 & -2.191572 & 0.142863 & 1.4259580\end{array}$




$\begin{array}{rrrr}1 & -2.091589 & 2.367507 & -1.0662450 \\ 1 & -3.146399 & -0.0164930 & -1.775946 \\ 1 & -3.133341 & -1.6117580 & 0.400890 \\ 1 & -1.394544 & 2.227266 & 1.5186040 \\ 1 & -2.027017 & -0.2206070 & 2.431475\end{array}$

3.

$\begin{array}{llll}22 & -0.582644 & -0.257085 & -0.222114\end{array}$

$\begin{array}{llll}6 & 2.912758 & 0.491296 & 0.912356\end{array}$

$\begin{array}{llll}6 & 1.063888 & 0.768710 & -1.263080\end{array}$

$\begin{array}{llll}6 & 1.564401 & 1.852508 & -0.565535\end{array}$

$\begin{array}{llll}6 & 2.530093 & 1.722363 & 0.486707\end{array}$

$\begin{array}{llll}6 & 2.235698 & -0.731611 & 0.415077\end{array}$

$\begin{array}{lllll}6 & 0.897627 & -1.124394 & 1.152208\end{array}$

$\begin{array}{lllll}6 & 0.312938 & -2.143327 & 0.331866\end{array}$

$\begin{array}{llll}6 & 0.591691 & -1.806894 & -1.035350\end{array}$

$\begin{array}{llll}6 & 1.662715 & -0.660126 & -1.036606\end{array}$

$\begin{array}{llll}1 & 3.673220 & 0.382229 & 1.682127\end{array}$

$\begin{array}{llll}1 & 0.569602 & 0.939365 & -2.227956\end{array}$

$\begin{array}{llll}1 & 1.264809 & 2.852076 & -0.875422\end{array}$

$\begin{array}{llll}1 & 2.962348 & 2.624988 & 0.911436\end{array}$

$\begin{array}{llll}1 & 2.924017 & -1.585981 & 0.490350\end{array}$

$\begin{array}{llll}1 & 0.860085 & -1.159066 & 2.237975\end{array}$

$\begin{array}{llll}1 & -0.250339 & -3.011709 & 0.657983\end{array}$

$\begin{array}{llll}1 & 0.467933 & -2.460302 & -1.891047\end{array}$

$\begin{array}{llll}1 & 2.380311 & -0.833618 & -1.849301\end{array}$

$\begin{array}{lllll}6 & -2.384342 & 1.266607 & -0.671475\end{array}$

$\begin{array}{lllll}6 & -2.837223 & -0.089444 & -0.704818\end{array}$

$\begin{array}{llll}6 & -2.712194 & -0.619720 & 0.614243\end{array}$

$\begin{array}{llll}6 & -1.990773 & 1.565102 & 0.652219\end{array}$

$\begin{array}{llll}6 & -2.172273 & 0.404623 & 1.446764\end{array}$

$\begin{array}{llll}1 & -2.347017 & 1.945773 & -1.514819\end{array}$

$\begin{array}{llll}1 & -3.242381 & -0.607648 & -1.565422\end{array}$

$\begin{array}{llll}1 & -2.995449 & -1.614768 & 0.932342\end{array}$

$\begin{array}{llll}1 & -1.556511 & 2.498051 & 0.990679\end{array}$

$\begin{array}{llll}1 & -1.942478 & 0.312315 & 2.500130\end{array}$

TS3.

$\begin{array}{lrrr}22 & -0.427856 & 0.162736 & -0.111025 \\ 6 & 3.151716 & -0.532694 & -0.370857 \\ 6 & 0.815110 & -0.940727 & 1.201150 \\ 6 & 1.092395 & -1.727611 & 0.030349 \\ 6 & 2.427235 & -1.657059 & -0.543409 \\ 6 & 2.399086 & 0.662344 & 0.1283010 \\ 6 & 1.392113 & 1.108891 & -0.9784630\end{array}$




$\begin{array}{rrrr}6 & 0.580268 & 2.185050 & -0.5150510 \\ 6 & 0.061052 & 1.900106 & 0.7453420 \\ 6 & 1.438764 & 0.361031 & 1.2988460 \\ 1 & 4.179791 & -0.423434 & -0.701796 \\ 1 & 0.273124 & -1.342120 & 2.054191 \\ 1 & 0.558764 & -2.670571 & -0.058499 \\ 1 & 2.826652 & -2.532176 & -1.053364 \\ 1 & 3.075959 & 1.488569 & 0.3821830 \\ 1 & 1.710602 & 1.036988 & -2.0170660 \\ 1 & 0.307407 & 3.062295 & -1.1034530 \\ 1 & -0.341727 & 2.593445 & 1.478899 \\ 1 & 1.611358 & 0.780893 & 2.2862140 \\ 6 & -2.188223 & -1.435811 & 0.419847 \\ 6 & -2.565155 & -0.224474 & 1.048194 \\ 6 & -2.750988 & 0.757511 & 0.044110 \\ 6 & -2.143311 & -1.210531 & -0.982847 \\ 6 & -2.491808 & 0.149955 & -1.215826 \\ 1 & -1.962180 & -2.369642 & 0.917957 \\ 1 & -2.658855 & -0.063177 & 2.115567 \\ 1 & -3.032272 & 1.789851 & 0.204289 \\ 1 & -1.890063 & -1.943142 & -1.738373 \\ 1 & -2.555241 & 0.636139 & -2.182335\end{array}$

4.

$\begin{array}{llll}22 & -0.410808 & 0.219281 & 0.019499\end{array}$

$\begin{array}{lllll}6 & 3.121146 & -0.341661 & -0.4677380\end{array}$

$\begin{array}{llll}6 & 0.999663 & -1.111247 & 1.2185380\end{array}$

$\begin{array}{llll}6 & 1.189827 & -1.736555 & -0.0465690\end{array}$

$\begin{array}{llll}6 & 2.467463 & -1.495243 & -0.7097590\end{array}$

$\begin{array}{llll}6 & 2.312100 & 0.7315070 & 0.210458\end{array}$

$\begin{array}{llll}6 & 1.262916 & 1.2260860 & -0.859589\end{array}$

$\begin{array}{llll}6 & 0.393700 & 2.3019050 & -0.458934\end{array}$

$\begin{array}{llll}6 & -0.370196 & 2.084748 & 0.672314\end{array}$

$\begin{array}{llll}6 & 1.540584 & 0.1624480 & 1.402732\end{array}$

$\begin{array}{llll}1 & 4.119351 & -0.124134 & -0.8350200\end{array}$

$\begin{array}{lllll}1 & 0.432703 & -1.591621 & 2.0132080\end{array}$

$\begin{array}{lllll}1 & 0.689731 & -2.683468 & -0.2258330\end{array}$

$\begin{array}{llll}1 & 2.891267 & -2.285720 & -1.3262330\end{array}$

$\begin{array}{llll}1 & 2.928061 & 1.5804040 & 0.529024\end{array}$

$\begin{array}{llll}1 & 1.623199 & 1.1982750 & -1.886299\end{array}$

$\begin{array}{llll}1 & 0.196238 & 3.1342760 & -1.143675\end{array}$

$\begin{array}{llll}1 & -1.019164 & 2.841482 & 1.108684\end{array}$

$\begin{array}{llll}1 & 1.578984 & 0.6401430 & 2.374269\end{array}$

$\begin{array}{llll}6 & -2.102980 & -1.477870 & 0.510272\end{array}$

$\begin{array}{llll}6 & -2.621807 & -0.230509 & 0.940201\end{array}$

$\begin{array}{llll}6 & -2.755169 & 0.608294 & -0.195105\end{array}$ 


$\begin{array}{lrrr}6 & -1.914259 & -1.415539 & -0.895748 \\ 6 & -2.313587 & -0.125466 & -1.331979 \\ 1 & -1.881428 & -2.328292 & 1.141938 \\ 1 & -2.846937 & 0.049640 & 1.961935 \\ 1 & -3.127322 & 1.623241 & -0.199730 \\ 1 & -1.522734 & -2.203731 & -1.524102 \\ 1 & -2.280587 & 0.239928 & -2.351708\end{array}$

\section{TS4}

$\begin{array}{llll}22 & 0.580374 & 0.342885 & -0.059169\end{array}$

$\begin{array}{llll}6 & -2.852315 & 0.659072 & 0.569213\end{array}$

$\begin{array}{llll}6 & -1.667648 & -1.299711 & -1.066174\end{array}$

$\begin{array}{llll}6 & -2.503361 & -1.669371 & -0.042476\end{array}$

$\begin{array}{llll}6 & -3.139291 & -0.659676 & 0.752371\end{array}$

$\begin{array}{llll}6 & -1.798103 & 1.079517 & -0.343124\end{array}$

$\begin{array}{llll}6 & -0.330624 & 1.736776 & 0.894852\end{array}$

$\begin{array}{llll}6 & 0.616736 & 2.528089 & 0.086622\end{array}$

$\begin{array}{llll}6 & 1.287317 & 1.930339 & -0.965064\end{array}$

$\begin{array}{llll}6 & -1.280736 & 0.067755 & -1.283940\end{array}$

$\begin{array}{llll}1 & -3.386002 & 1.424917 & 1.126722\end{array}$

$\begin{array}{llll}1 & -1.297681 & -2.051362 & -1.759178\end{array}$

$\begin{array}{llll}1 & -2.764830 & -2.713571 & 0.104169\end{array}$

$\begin{array}{llll}1 & -3.899271 & -0.955250 & 1.471995\end{array}$

$\begin{array}{llll}1 & -1.889591 & 2.080096 & -0.757684\end{array}$

$\begin{array}{llll}1 & -0.861387 & 2.222392 & 1.705273\end{array}$

$\begin{array}{llll}1 & 0.832839 & 3.547458 & 0.420904\end{array}$

$\begin{array}{llll}1 & 2.099464 & 2.435768 & -1.479521\end{array}$

$\begin{array}{llll}1 & -1.013286 & 0.373339 & -2.290064\end{array}$

$\begin{array}{llll}6 & 1.511396 & -1.869099 & -0.525405\end{array}$

$\begin{array}{llll}6 & 2.563842 & -0.946351 & -0.721369\end{array}$

$\begin{array}{llll}6 & 2.817754 & -0.301571 & 0.518355\end{array}$

$\begin{array}{llll}6 & 1.107335 & -1.794650 & 0.836887\end{array}$

$\begin{array}{llll}6 & 1.925006 & -0.832998 & 1.487317\end{array}$

$\begin{array}{llll}1 & 1.079771 & -2.510450 & -1.282167\end{array}$

$\begin{array}{llll}1 & 3.064975 & -0.736677 & -1.657297\end{array}$

$\begin{array}{llll}1 & 3.550300 & 0.476942 & 0.688314\end{array}$

$\begin{array}{llll}1 & 0.306636 & -2.361979 & 1.293498\end{array}$

$\begin{array}{llll}1 & 1.865982 & -0.543822 & 2.528352\end{array}$

5.

$\begin{array}{llll}22 & 0.7642360 & 0.427532 & 0.013332\end{array}$

$\begin{array}{llll}6 & 0.1131030 & 1.508224 & 1.421691\end{array}$

$\begin{array}{llll}6 & 0.3630360 & 2.455572 & 0.370700\end{array}$

$\begin{array}{llll}6 & 0.8490740 & 2.063820 & -0.927467\end{array}$

$\begin{array}{llll}1 & -0.1191670 & 1.884003 & 2.413751\end{array}$

$\begin{array}{llll}1 & 0.2301120 & 3.518843 & 0.579290\end{array}$ 


$\begin{array}{rrrl}1 & 1.1637680 & 2.842781 & -1.616328 \\ 6 & -3.2456040 & 0.739512 & -0.0991300 \\ 6 & -1.7887820 & -1.634508 & -0.346670 \\ 6 & -2.7767130 & -1.521134 & 0.6340560 \\ 6 & -3.5059890 & -0.336837 & 0.7526180 \\ 6 & -2.2619080 & 0.629782 & -1.0835630 \\ 6 & -1.5290790 & -0.559643 & -1.211940 \\ 1 & -3.8121230 & 1.661085 & 0.000190 \\ 1 & -1.2390790 & -2.564227 & -0.457310 \\ 1 & -2.9795910 & -2.355687 & 1.2993920 \\ 1 & -4.2749540 & -0.249251 & 1.5156230 \\ 1 & -2.0539940 & 1.459856 & -1.7504980 \\ 1 & -0.8207240 & -0.670521 & -2.030460 \\ 6 & 1.6795480 & -1.752736 & -0.6440170 \\ 6 & 2.6045090 & -0.773054 & -1.0676940 \\ 6 & 3.0736510 & -0.094136 & 0.088918 \\ 6 & 1.5874150 & -1.703903 & 0.777652 \\ 6 & 2.4607390 & -0.683933 & 1.231212 \\ 1 & 1.1340110 & -2.427184 & -1.2934570 \\ 1 & 2.8696820 & -0.540813 & -2.0907730 \\ 1 & 3.7766400 & 0.728973 & 0.097653 \\ 1 & 0.9590760 & -2.326535 & 1.401224 \\ 1 & 2.6151580 & -0.389195 & 2.260266\end{array}$

6.

$\begin{array}{lrrr}22 & 0.338199 & 0.407913 & -0.000417 \\ 6 & 0.216897 & 1.820085 & 1.261689 \\ 6 & 0.156471 & 2.510698 & 0.001684 \\ 6 & 0.208248 & 1.821747 & -1.259742 \\ 1 & 0.300527 & 2.411340 & 2.169163 \\ 1 & 0.116796 & 3.602304 & 0.002585 \\ 1 & 0.286664 & 2.414564 & -2.166665 \\ 6 & 0.730519 & -2.021862 & 0.039035 \\ 6 & 1.324244 & -1.501520 & -1.139833 \\ 6 & 2.348902 & -0.602040 & -0.746409 \\ 6 & 1.387610 & -1.458560 & 1.161461 \\ 6 & 2.386569 & -0.573724 & 0.677828 \\ 1 & -0.099834 & -2.716275 & 0.07523 \\ 1 & 1.028475 & -1.723750 & -2.157069 \\ 1 & 2.983081 & -0.031587 & -1.412096 \\ 1 & 1.146990 & -1.639092 & 2.201170 \\ 1 & 3.054486 & 0.022318 & 1.285802 \\ 6 & -2.854245 & -0.460328 & 0.00239 \\ 6 & -4.280985 & -0.756045 & 0.00037 \\ 7 & -1.721956 & -0.211911 & 0.00323 \\ 1 & -4.766223 & -0.267047 & -0.85089\end{array}$




$\begin{array}{cccc}1 & -4.439091 & -1.836436 & -0.07749 \\ 1 & -4.743928 & -0.397742 & 0.92590\end{array}$

\section{TS5}

$\begin{array}{lrrr}22 & 0.145134 & 0.2897400 & -0.093984 \\ 6 & -0.926216 & 1.216203 & 1.176839 \\ 6 & -0.830319 & 2.152772 & 0.080474 \\ 6 & -0.142425 & 1.848191 & -1.135404 \\ 1 & -1.328741 & 1.581104 & 2.118335 \\ 1 & -1.241979 & 3.155138 & 0.218805 \\ 1 & 0.027465 & 2.6396100 & -1.859094 \\ 6 & 1.555633 & -1.7067790 & -0.241742 \\ 6 & 2.164322 & -0.6867510 & -1.013038 \\ 6 & 2.503335 & 0.3766260 & -0.134398 \\ 6 & 1.524313 & -1.2897720 & 1.113617 \\ 6 & 2.109157 & 0.0025260 & 1.182741 \\ 1 & 1.145835 & -2.6311850 & -0.629062 \\ 1 & 2.304496 & -0.6950210 & -2.086056 \\ 1 & 2.975713 & 1.3079730 & -0.417256 \\ 1 & 1.103227 & -1.8422480 & 1.944020 \\ 1 & 2.226919 & 0.5995520 & 2.077384 \\ 6 & -2.397676 & -0.988499 & -0.377487 \\ 6 & -3.652625 & -0.928735 & 0.359433 \\ 7 & -1.421560 & -1.104696 & -1.002262 \\ 1 & -4.235727 & -1.838393 & 0.180483 \\ 1 & -3.430788 & -0.835334 & 1.426207 \\ 1 & -4.233450 & -0.057315 & 0.043532\end{array}$

$\begin{array}{lrrr}7 . & & & \\ 22 & -0.119023 & 0.103190 & -0.265080 \\ 6 & 0.216296 & 1.990133 & -0.668080 \\ 6 & 1.183288 & 1.940970 & 0.319920 \\ 6 & 1.876085 & 0.739853 & 0.718920 \\ 6 & 2.099892 & -0.404185 & -0.128080 \\ 7 & 1.293857 & -0.608049 & -1.160080 \\ 6 & 3.201726 & -1.384371 & 0.226920 \\ 1 & -0.245543 & 2.941212 & -0.923080 \\ 1 & 1.365434 & 2.804939 & 0.964920 \\ 1 & 2.377089 & 0.760768 & 1.682920 \\ 1 & 3.401724 & -1.397405 & 1.303920 \\ 1 & 4.128776 & -1.087528 & -0.280080 \\ 1 & 2.941556 & -2.391327 & -0.109080 \\ 6 & -2.155192 & -0.902466 & -0.884080 \\ 6 & -1.656315 & -1.627550 & 0.224920 \\ 6 & -1.698170 & -0.767543 & 1.360920 \\ 6 & -2.515972 & 0.400595 & -0.438080\end{array}$




$\begin{array}{rrrr}6 & -2.251960 & 0.473550 & 0.952920 \\ 1 & -2.220254 & -1.266455 & -1.903080 \\ 1 & -1.284487 & -2.644613 & 0.204920 \\ 1 & -1.382213 & -1.025597 & 2.364920 \\ 1 & -2.921838 & 1.194664 & -1.053080 \\ 1 & -2.396813 & 1.343575 & 1.581920\end{array}$

8.

$\begin{array}{llll}22 & -0.580420 & 0.140320 & -0.16711\end{array}$

$\begin{array}{llll}6 & 0.423931 & 0.371644 & 1.622473\end{array}$

$\begin{array}{lllll}6 & 1.678495 & -0.142133 & 1.905683\end{array}$

$\begin{array}{llll}6 & 2.500305 & -0.871058 & 1.002614\end{array}$

$\begin{array}{llll}6 & 2.091398 & -1.142775 & -0.306840\end{array}$

$\begin{array}{llll}7 & 0.887961 & -0.712637 & -0.682665\end{array}$

$\begin{array}{lllll}6 & 2.972716 & -1.884669 & -1.289569\end{array}$

$\begin{array}{lllll}6 & 0.758457 & 2.130653 & -0.539224\end{array}$

$\begin{array}{llll}6 & 2.170720 & 2.416662 & -0.308297\end{array}$

$\begin{array}{llll}7 & -0.369294 & 2.173765 & -0.894858\end{array}$

$\begin{array}{llll}1 & -0.025147 & 0.940872 & 2.448229\end{array}$

$\begin{array}{llll}1 & 2.111415 & 0.025961 & 2.896691\end{array}$

$\begin{array}{llll}1 & 3.475691 & -1.218246 & 1.336720\end{array}$

$\begin{array}{llll}1 & 3.940229 & -2.147379 & -0.849529\end{array}$

$\begin{array}{llll}1 & 3.148379 & -1.278008 & -2.186326\end{array}$

$\begin{array}{llll}1 & 2.479905 & -2.806517 & -1.621232\end{array}$

$\begin{array}{llll}1 & 2.714093 & 1.481281 & -0.130922\end{array}$

$\begin{array}{llll}1 & 2.585621 & 2.935123 & -1.179854\end{array}$

$\begin{array}{llll}1 & 2.269212 & 3.050321 & 0.578704\end{array}$

$\begin{array}{llll}6 & -2.967006 & 0.242616 & -0.773297\end{array}$

$\begin{array}{lllll}6 & -2.444631 & -1.042991 & -1.074623\end{array}$

$\begin{array}{llll}6 & -2.068207 & -1.663020 & 0.146446\end{array}$

$\begin{array}{llll}6 & -2.914757 & 0.416776 & 0.631280\end{array}$

$\begin{array}{llll}6 & -2.353627 & -0.755083 & 1.201090\end{array}$

$\begin{array}{llll}1 & -3.310843 & 0.974723 & -1.493024\end{array}$

$\begin{array}{llll}1 & -2.331279 & -1.469160 & -2.063862\end{array}$

$\begin{array}{llll}1 & -1.623092 & -2.643534 & 0.252825\end{array}$

$\begin{array}{llll}1 & -3.210867 & 1.308014 & 1.1715650\end{array}$

$\begin{array}{llll}1 & -2.171476 & -0.928311 & 2.2528470\end{array}$

$\begin{array}{llll}\text { TS6 } & & & \\ 22 & -0.575947 & 0.107824 & -0.2079120 \\ 6 & 0.487479 & 0.579604 & 1.489639 \\ 6 & 1.735472 & 0.061548 & 1.828520 \\ 6 & 2.522001 & -0.785583 & 1.0183020 \\ 6 & 2.086541 & -1.201329 & -0.2528020 \\ 7 & 0.881963 & -0.822372 & -0.6511540 \\ 6 & 2.960822 & -2.042792 & -1.1597940\end{array}$




$\begin{array}{lrrl}6 & 0.698656 & 2.061411 & -0.589501 \\ 6 & 2.074593 & 2.527664 & -0.3886600 \\ 7 & -0.384899 & 2.039815 & -1.0849570 \\ 1 & 0.074891 & 1.278405 & 2.230010 \\ 1 & 2.178941 & 0.338683 & 2.789465 \\ 1 & 3.499874 & -1.103317 & 1.3731740 \\ 1 & 3.939023 & -2.243434 & -0.7109590 \\ 1 & 3.112324 & -1.539120 & -2.1219440 \\ 1 & 2.473234 & -3.001089 & -1.3749190 \\ 1 & 2.739371 & 1.666697 & -0.2687420 \\ 1 & 2.385273 & 3.130273 & -1.2497030 \\ 1 & 2.126709 & 3.129395 & 0.523657 \\ 6 & -2.969148 & 0.120847 & -0.7842650 \\ 6 & -2.451950 & -1.195033 & -0.914510 \\ 6 & -2.054111 & -1.642186 & 0.3729230 \\ 6 & -2.893671 & 0.485879 & 0.5823530 \\ 6 & -2.321779 & -0.597543 & 1.2981290 \\ 1 & -3.324143 & 0.748513 & -1.5917930 \\ 1 & -2.354167 & -1.752455 & -1.837960 \\ 1 & -1.606006 & -2.599597 & 0.6042160 \\ 1 & -3.180253 & 1.443747 & 0.9995980 \\ 1 & -2.123115 & -0.625861 & 2.3608020\end{array}$

$\begin{array}{lccc}\text { 9. } & & & \\ 22 & -0.613941 & -0.004947 & -0.378450 \\ 6 & 0.612710 & 1.082728 & 1.026630 \\ 6 & 1.758236 & 0.411312 & 1.6209250 \\ 6 & 2.447704 & -0.634343 & 1.0728700 \\ 6 & 2.005238 & -1.336999 & -0.1262360 \\ 7 & 0.820398 & -1.142401 & -0.6014200 \\ 6 & 2.972348 & -2.300090 & -0.7878650 \\ 6 & 0.794481 & 1.806967 & -0.3353460 \\ 6 & 2.058641 & 2.593631 & -0.6009850 \\ 7 & -0.142560 & 1.659475 & -1.1965210 \\ 1 & 0.077741 & 1.744923 & 1.711049 \\ 1 & 2.112899 & 0.7809010 & 2.5853360 \\ 1 & 3.365920 & -0.975858 & 1.5467430 \\ 1 & 3.346809 & -3.031495 & -0.0603330 \\ 1 & 3.844534 & -1.761599 & -1.1805800 \\ 1 & 2.482419 & -2.827701 & -1.6085990 \\ 1 & 2.935727 & 1.960718 & -0.431183 \\ 1 & 2.062209 & 2.969632 & -1.626980 \\ 1 & 2.125276 & 3.436338 & 0.099107 \\ 6 & -3.019925 & 0.082066 & -0.73534 \\ 6 & -2.614369 & -1.27792 & -0.623299\end{array}$




$\begin{array}{lllr}6 & -2.147523 & -1.48876 & 0.700338 \\ 6 & -2.785289 & 0.713127 & 0.50764 \\ 6 & -2.232654 & -0.25334 & 1.393013 \\ 1 & -3.395731 & 0.562055 & -1.63057 \\ 1 & -2.652414 & -2.02200 & -1.409736 \\ 1 & -1.749131 & -2.41503 & 1.094278 \\ 1 & -2.954382 & 1.759157 & 0.72956 \\ 1 & -1.937624 & -0.08090 & 2.419471\end{array}$

\section{TS7.}

$\begin{array}{lccc}22 & 0.826067 & -0.162004 & -0.27705 \\ 6 & -1.990027 & -1.45141 & 0.937349 \\ 6 & -2.910454 & -0.53329 & 1.304115 \\ 6 & -3.012279 & 0.763199 & 0.67738 \\ 6 & -1.990757 & 1.260502 & -0.08226 \\ 7 & -0.866730 & 0.521884 & -0.35878 \\ 6 & -1.969693 & 2.679531 & -0.59382 \\ 6 & -1.124419 & -1.26757 & -0.274552 \\ 6 & -1.824443 & -1.57731 & -1.596253 \\ 7 & 0.185682 & -1.803946 & -0.16833 \\ 1 & -1.874754 & -2.39322 & 1.466768 \\ 1 & -3.575342 & -0.74830 & 2.138568 \\ 1 & -3.857458 & 1.404033 & 0.90848 \\ 1 & -2.862623 & 3.227368 & -0.27833 \\ 1 & -1.913052 & 2.701595 & -1.68921 \\ 1 & -1.084739 & 3.207681 & -0.21536 \\ 1 & -2.754468 & -1.00966 & -1.694676 \\ 1 & -1.166421 & -1.34672 & -2.442523 \\ 1 & -2.057985 & -2.64817 & -1.627798 \\ 6 & 3.182854 & -0.691024 & -0.21801 \\ 6 & 3.047487 & 0.594274 & -0.822771 \\ 6 & 2.545906 & 1.493592 & 0.153678 \\ 6 & 2.729780 & -0.592227 & 1.11624 \\ 6 & 2.316456 & 0.755370 & 1.344216 \\ 1 & 3.523089 & -1.594212 & -0.70832 \\ 1 & 3.310104 & 0.848996 & -1.843481 \\ 1 & 2.324326 & 2.542389 & 0.000455 \\ 1 & 2.663562 & -1.404389 & 1.82751 \\ 1 & 1.917164 & 1.149466 & 2.271012\end{array}$

10.

$\begin{array}{llll}22 & 0.857034 & -0.2946280 & -0.358851\end{array}$

$\begin{array}{llll}6 & -2.070590 & -1.3090350 & 0.987774\end{array}$

$\begin{array}{llll}6 & -2.903110 & -0.3209450 & 1.374808\end{array}$

$\begin{array}{llll}6 & -2.905629 & 0.9640460 & 0.705985\end{array}$

$\begin{array}{llll}6 & -1.888800 & 1.2901060 & -0.140651\end{array}$ 


$\begin{array}{lrcr}7 & -0.903191 & 0.3669720 & -0.439749 \\ 6 & -1.703851 & 2.6612700 & -0.731716 \\ 6 & -1.231081 & -1.1453270 & -0.253603 \\ 6 & -1.947482 & -1.6522480 & -1.512038 \\ 7 & 0.097722 & -1.8350120 & -0.121626 \\ 1 & -2.018449 & -2.2677100 & 1.494854 \\ 1 & -3.566524 & -0.4650890 & 2.224922 \\ 1 & -3.656320 & 1.7066710 & 0.956531 \\ 1 & -2.499957 & 3.3398980 & -0.411816 \\ 1 & -1.696956 & 2.6221020 & -1.828306 \\ 1 & -0.741377 & 3.0890120 & -0.419733 \\ 1 & -2.891580 & -1.1159720 & -1.660286 \\ 1 & -1.311819 & -1.5058180 & -2.394441 \\ 1 & -2.155362 & -2.7225610 & -1.409997 \\ 6 & 3.181655 & -0.7480450 & 0.026901 \\ 6 & 3.142507 & 0.439345 & -0.7608630 \\ 6 & 2.544400 & 1.465913 & 0.0144510 \\ 6 & 2.576360 & -0.4646360 & 1.273715 \\ 6 & 2.165863 & 0.902878 & 1.2618860 \\ 1 & 3.570063 & -1.7090140 & -0.286538 \\ 1 & 3.529952 & 0.546306 & -1.7681370 \\ 1 & 2.360863 & 2.483598 & -0.3078230 \\ 1 & 2.428982 & -1.167114 & 2.0830070 \\ 1 & 1.670568 & 1.423864 & 2.0722100\end{array}$

TS8.

$\begin{array}{lrlr}22 & 0.740777 & -0.543116 & -0.1935300 \\ 6 & -2.429550 & -0.9584250 & 0.901450 \\ 6 & -2.842894 & 0.2476000 & 1.376529 \\ 6 & -2.441141 & 1.465581 & 0.7398470 \\ 6 & -1.547600 & 1.4145690 & -0.294829 \\ 7 & -1.058743 & 0.2028550 & -0.765206 \\ 6 & -1.057279 & 2.6372840 & -1.020099 \\ 6 & -1.576527 & -1.0213420 & -0.276964 \\ 6 & -1.917286 & -2.0458040 & -1.339790 \\ 7 & 0.103540 & -1.9835950 & 0.347038 \\ 1 & -2.745113 & -1.8923410 & 1.354059 \\ 1 & -3.507639 & 0.2926350 & 2.235798 \\ 1 & -2.836749 & 2.4192210 & 1.072500 \\ 1 & -1.453234 & 3.5495470 & -0.565149 \\ 1 & -1.367291 & 2.6073780 & -2.071997 \\ 1 & 0.037479 & 2.691035 & -1.0111960 \\ 1 & -2.868812 & -1.7636770 & -1.812914 \\ 1 & -1.146808 & -2.0785400 & -2.116758 \\ 1 & -2.014085 & -3.0428600 & -0.903550 \\ 6 & 3.079276 & -0.1592580 & -0.698474\end{array}$




$\begin{array}{lllr}6 & 2.545084 & 1.156239 & -0.5755900 \\ 6 & 2.071888 & 1.307250 & 0.7483860 \\ 6 & 2.989823 & -0.7970970 & 0.568822 \\ 6 & 2.340646 & 0.094676 & 1.4558640 \\ 1 & 3.524247 & -0.5836870 & -1.591932 \\ 1 & 2.482409 & 1.891998 & -1.3681680 \\ 1 & 1.591071 & 2.186527 & 1.1604740 \\ 1 & 3.303871 & -1.806411 & 0.7994320 \\ 1 & 2.103335 & -0.1047430 & 2.493333\end{array}$

$\begin{array}{lccc}\text { 11. } & & & \\ 22 & 0.984911 & -0.000142 & 0.6242300 \\ 7 & 0.936301 & 0.000536 & 2.2361910 \\ 6 & -3.212488 & 1.2009570 & -0.297124 \\ 6 & -3.903399 & -0.0003800 & -0.438565 \\ 6 & -3.212116 & -1.2014920 & -0.296958 \\ 6 & -1.843556 & -1.1722590 & -0.027828 \\ 7 & -1.180558 & 0.0000930 & 0.089128 \\ 6 & -1.027112 & -2.4192440 & 0.176844 \\ 6 & -1.843906 & 1.1722260 & -0.027984 \\ 6 & -1.027918 & 2.4195250 & 0.176507 \\ 1 & -3.722103 & 2.1548190 & -0.384989 \\ 1 & -4.969444 & -0.0005570 & -0.647199 \\ 1 & -3.721433 & -2.1555270 & -0.384668 \\ 1 & -1.649420 & -3.3177520 & 0.156328 \\ 1 & -0.518738 & -2.3792660 & 1.151666 \\ 1 & -0.262783 & -2.5235360 & -0.605170 \\ 1 & -0.519433 & 2.3797910 & 1.151269 \\ 1 & -0.263657 & 2.5239930 & -0.605541 \\ 1 & -1.650590 & 3.3177820 & 0.155939 \\ 6 & 3.360742 & 0.000712 & 0.2718100 \\ 6 & 2.874344 & -1.144311 & -0.4094220 \\ 6 & 2.138536 & -0.7070230 & -1.551867 \\ 6 & 2.873460 & 1.144851 & -0.4102040 \\ 6 & 2.137928 & 0.706192 & -1.5523160 \\ 1 & 3.950621 & 0.001231 & 1.1779190 \\ 1 & 3.074693 & -2.1738390 & -0.134582 \\ 1 & 1.667645 & -1.3454010 & -2.290899 \\ 1 & 3.072790 & 2.174739 & -0.1359680 \\ 1 & 1.666531 & 1.343718 & -2.2917570\end{array}$

12.

$\begin{array}{llll}22 & 0.243967 & -0.4524820 & -0.064853\end{array}$

$\begin{array}{llll}6 & -1.421679 & -0.2857450 & -1.452445\end{array}$

$\begin{array}{llll}6 & -2.776661 & -0.3010000 & -0.917602\end{array}$

$\begin{array}{llll}6 & -3.139748 & 0.0987210 & 0.336041\end{array}$ 


$\begin{array}{rrrr}6 & -2.170492 & 0.4467010 & 1.374737 \\ 7 & -0.920874 & 0.1622370 & 1.236397 \\ 6 & -2.686225 & 1.1432310 & 2.620303 \\ 6 & -0.666001 & 1.0439680 & -1.570580 \\ 6 & -1.406402 & 2.312118 & -1.9385240 \\ 7 & 0.584733 & 1.009007 & -1.2785030 \\ 6 & 2.188032 & -1.8577750 & -0.287897 \\ 6 & 1.628496 & -2.0650630 & 1.003235 \\ 6 & 0.360559 & -2.6800560 & 0.836986 \\ 6 & 1.257634 & -2.3163950 & -1.249454 \\ 6 & 0.120604 & -2.8151700 & -0.552881 \\ 6 & 3.023057 & 1.909261 & 0.7961790 \\ 6 & 2.976739 & 3.027606 & -0.1398850 \\ 7 & 3.033747 & 0.999052 & 1.5175610 \\ 1 & -1.290206 & -0.8646560 & -2.368537 \\ 1 & -3.575648 & -0.6506500 & -1.574834 \\ 1 & -4.195569 & 0.1604220 & 0.593341 \\ 1 & -3.483538 & 0.5516790 & 3.088720 \\ 1 & -3.118081 & 2.120442 & 2.3680550 \\ 1 & -1.876091 & 1.2850980 & 3.338248 \\ 1 & -2.260460 & 2.4583300 & -1.269606 \\ 1 & -0.739529 & 3.1762850 & -1.881756 \\ 1 & -1.804753 & 2.2259020 & -2.957702 \\ 1 & 3.134218 & -1.3729020 & -0.491567 \\ 1 & 2.084155 & -1.7717880 & 1.939745 \\ 1 & -0.328064 & -2.9387400 & 1.630620 \\ 1 & 1.374474 & -2.2640320 & -2.324600 \\ 1 & -0.772434 & -3.2252880 & -1.005079 \\ 1 & 2.694612 & 3.948840 & 0.3796310 \\ 1 & 3.953269 & 3.171924 & -0.6128030 \\ 1 & 2.225658 & 2.789254 & -0.9025670\end{array}$

TS9.

$\begin{array}{lllr}22 & 0.612569 & -0.1708490 & 0.056068 \\ 6 & -0.833909 & -0.2133030 & -1.654612 \\ 6 & -2.076640 & -0.9764210 & -1.531205 \\ 6 & -2.716864 & -1.2113920 & -0.357891 \\ 6 & -2.148556 & -0.8385410 & 0.951060 \\ 7 & -0.905778 & -0.5535270 & 1.091629 \\ 6 & -3.098823 & -0.8217170 & 2.135165 \\ 6 & -0.751390 & 1.2073240 & -1.260708 \\ 6 & -1.972840 & 2.0860090 & -1.060412 \\ 7 & 0.458743 & 1.591723 & -0.9633730 \\ 6 & 2.872656 & -0.9649340 & 0.230235 \\ 6 & 2.081420 & -1.7392030 & 1.114581 \\ 6 & 1.165907 & -2.4944120 & 0.336782\end{array}$




$\begin{array}{cccc}6 & 2.440208 & -1.2269750 & -1.094561 \\ 6 & 1.384210 & -2.1756790 & -1.025774 \\ 6 & 1.092536 & 2.390928 & 0.7162260 \\ 6 & 0.944363 & 3.850156 & 0.5624410 \\ 7 & 1.447005 & 1.442225 & 1.3460520 \\ 1 & -0.235982 & -0.4216850 & -2.539964 \\ 1 & -2.527144 & -1.3768200 & -2.442010 \\ 1 & -3.692578 & -1.6946860 & -0.360243 \\ 1 & -3.563541 & -1.8081230 & 2.266378 \\ 1 & -3.914128 & -0.1050530 & 1.970362 \\ 1 & -2.562415 & -0.5546900 & 3.047810 \\ 1 & -2.237189 & 2.1446230 & 0.002922 \\ 1 & -1.742265 & 3.1000680 & -1.403614 \\ 1 & -2.842942 & 1.7093660 & -1.605020 \\ 1 & 3.626774 & -0.2465290 & 0.522472 \\ 1 & 2.129764 & -1.711867 & 2.1952140 \\ 1 & 0.405983 & -3.162117 & 0.7189290 \\ 1 & 2.830004 & -0.7669630 & -1.993530 \\ 1 & 0.838714 & -2.5842380 & -1.864964 \\ 1 & -0.112663 & 4.115522 & 0.4706360 \\ 1 & 1.371523 & 4.350940 & 1.4384620 \\ 1 & 1.458100 & 4.176930 & -0.3454690\end{array}$

$\begin{array}{lccc}\text { 13. } & & & \\ 22 & -0.582221 & -0.2583930 & -0.110184 \\ 6 & 0.879290 & 0.188552 & 1.7001000 \\ 6 & 2.207662 & -0.4397160 & 1.695187 \\ 6 & 2.858074 & -0.8243970 & 0.574712 \\ 6 & 2.284842 & -0.7423010 & -0.798277 \\ 7 & 1.029996 & -0.657114 & -1.0255540 \\ 6 & 3.289136 & -0.7843230 & -1.936793 \\ 6 & 0.588216 & 1.426578 & 1.0642140 \\ 6 & 1.637857 & 2.356930 & 0.5043340 \\ 7 & -0.719742 & 1.5775390 & 0.793183 \\ 6 & -2.767122 & -1.2192610 & -0.225530 \\ 6 & -1.924581 & -1.9844460 & -1.067278 \\ 6 & -0.954828 & -2.6304310 & -0.253712 \\ 6 & -2.307955 & -1.3726850 & 1.109026 \\ 6 & -1.190163 & -2.2517750 & 1.088790 \\ 6 & -1.227658 & 2.1279330 & -0.478370 \\ 6 & -1.449777 & 3.6081490 & -0.617387 \\ 7 & -1.490777 & 1.1946520 & -1.288896 \\ 1 & 0.177420 & -0.117078 & 2.4736220 \\ 1 & 2.697767 & -0.6138150 & 2.655188 \\ 1 & 3.871209 & -1.2158350 & 0.656727 \\ 1 & 3.871659 & -1.7149220 & -1.899473\end{array}$




$\begin{array}{cccc}1 & 4.006589 & 0.042909 & -1.8539630 \\ 1 & 2.773369 & -0.7219310 & -2.896834 \\ 1 & 2.645023 & 2.043878 & 0.7836660 \\ 1 & 1.576264 & 2.414428 & -0.5888210 \\ 1 & 1.460771 & 3.364248 & 0.8992880 \\ 1 & -3.569520 & -0.5734280 & -0.553769 \\ 1 & -1.973984 & -2.0194210 & -2.147733 \\ 1 & -0.151698 & -3.2628940 & -0.605749 \\ 1 & -2.723902 & -0.8876820 & 1.982945 \\ 1 & -0.603779 & -2.5590180 & 1.944866 \\ 1 & -0.507596 & 4.1632030 & -0.530376 \\ 1 & -1.905692 & 3.8234600 & -1.587000 \\ 1 & -2.109332 & 3.9601500 & 0.186244\end{array}$

TS10.

$\begin{array}{lllr}22 & -0.752609 & -0.2011240 & -0.181604 \\ 6 & 1.869358 & 0.793407 & 1.6105930 \\ 6 & 2.909532 & -0.0657020 & 1.514279 \\ 6 & 3.180277 & -0.8535130 & 0.335635 \\ 6 & 2.213090 & -1.0736790 & -0.611294 \\ 7 & 1.000021 & -0.4464960 & -0.581893 \\ 6 & 2.387214 & -2.0843140 & -1.720447 \\ 6 & 1.076594 & 1.201158 & 0.4155400 \\ 6 & 1.823988 & 2.045678 & -0.6114540 \\ 7 & -0.249876 & 1.5272500 & 0.698754 \\ 6 & -1.031524 & 2.0567450 & -0.451455 \\ 6 & -1.378462 & 3.5191650 & -0.361349 \\ 7 & -1.452760 & 1.2295070 & -1.331820 \\ 1 & 1.600372 & 1.272451 & 2.5478020 \\ 1 & 3.530857 & -0.2421090 & 2.390762 \\ 1 & 4.110153 & -1.409406 & 0.2700040 \\ 1 & 3.365985 & -2.5694640 & -1.661905 \\ 1 & 2.285811 & -1.605728 & -2.7015870 \\ 1 & 1.610179 & -2.8566450 & -1.657974 \\ 1 & 2.803099 & 1.611314 & -0.8203500 \\ 1 & 1.264340 & 2.136373 & -1.5453220 \\ 1 & 1.975467 & 3.044890 & -0.1832900 \\ 1 & -0.466815 & 4.1287180 & -0.346162 \\ 1 & -2.003627 & 3.8075050 & -1.209619 \\ 1 & -1.910410 & 3.7128670 & 0.577938 \\ 6 & -3.035284 & -0.7907910 & 0.386493 \\ 6 & -2.613275 & -1.6619030 & -0.648220 \\ 6 & -1.551477 & -2.4620880 & -0.144696 \\ 6 & -2.220685 & -1.0305080 & 1.522313 \\ 6 & -1.308681 & -2.0736870 & 1.195517 \\ 1 & -3.798664 & -0.0291310 & 0.297696\end{array}$




$\begin{array}{llll}1 & -3.006010 & -1.6831850 & -1.656885 \\ 1 & -1.004655 & -3.2156530 & -0.697034 \\ 1 & -2.270692 & -0.5026190 & 2.465803 \\ 1 & -0.533678 & -2.4670820 & 1.841392\end{array}$

$\begin{array}{lclr}\text { 14. } & & & \\ 22 & -0.853758 & 0.0432360 & 0.014043 \\ 6 & 2.257486 & 0.579714 & 1.4019780 \\ 6 & 3.062981 & -0.4993060 & 1.348124 \\ 6 & 2.972859 & -1.4376960 & 0.244285 \\ 6 & 1.889118 & -1.400809 & -0.5813250 \\ 7 & 0.944143 & -0.3942740 & -0.433101 \\ 6 & 1.611216 & -2.433133 & -1.6386880 \\ 6 & 1.387116 & 0.897732 & 0.2075270 \\ 6 & 2.136634 & 1.754329 & -0.8281510 \\ 7 & 0.123071 & 1.553614 & 0.6907030 \\ 6 & -0.686016 & 2.2306270 & -0.360505 \\ 6 & -0.777418 & 3.7271770 & -0.230510 \\ 7 & -1.350058 & 1.5072550 & -1.188179 \\ 1 & 2.245437 & 1.279220 & 2.2320100 \\ 1 & 3.761527 & -0.7077860 & 2.155520 \\ 1 & 3.708450 & -2.2282820 & 0.137538 \\ 1 & 2.387959 & -3.2031780 & -1.649322 \\ 1 & 1.558287 & -1.9720200 & -2.632837 \\ 1 & 0.644899 & -2.9224240 & -1.463410 \\ 1 & 3.034289 & 1.218008 & -1.1499650 \\ 1 & 1.515453 & 1.950238 & -1.7080100 \\ 1 & 2.440373 & 2.705415 & -0.3769290 \\ 1 & 0.213113 & 4.173880 & -0.3777490 \\ 1 & -1.480426 & 4.1318340 & -0.962385 \\ 1 & -1.096882 & 3.9839350 & 0.786225 \\ 6 & -2.995868 & -0.5002940 & 0.861402 \\ 6 & -3.025127 & -0.9511580 & -0.485358 \\ 6 & -2.095342 & -2.0106630 & -0.610206 \\ 6 & -2.037274 & -1.2780410 & 1.565739 \\ 6 & -1.475125 & -2.2091560 & 0.650148 \\ 1 & -3.580724 & 0.3149360 & 1.270633 \\ 1 & -3.615366 & -0.5212470 & -1.283103 \\ 1 & -1.861714 & -2.5410100 & -1.525117 \\ 1 & -1.767077 & -1.1686180 & 2.609121 \\ 1 & -0.696436 & -2.9262140 & 0.876107\end{array}$

TS11.

$\begin{array}{llll}22 & -0.700662 & -0.2259850 & -0.078227\end{array}$

$\begin{array}{llll}6 & 1.893262 & 1.494477 & 1.2460750\end{array}$

$\begin{array}{llll}6 & 2.890091 & 0.582844 & 1.3953220\end{array}$ 


$\begin{array}{llll}6 & 3.116020 & -0.435764 & 0.4133020 \\ 6 & 2.229355 & -0.564119 & -0.6206020 \\ 7 & 1.151542 & 0.300433 & -0.7700930 \\ 6 & 2.390541 & -1.5812440 & -1.717329 \\ 6 & 1.052847 & 1.461106 & 0.0487430 \\ 6 & 0.924836 & 2.754831 & -0.7359070 \\ 7 & -0.732900 & 1.3758450 & 0.772613 \\ 6 & -1.787648 & 1.6092600 & -0.292789 \\ 6 & -2.607260 & 2.8497760 & -0.062992 \\ 7 & -1.967904 & 0.7360850 & -1.215651 \\ 1 & 1.721623 & 2.286593 & 1.9671330 \\ 1 & 3.546953 & 0.640630 & 2.2602650 \\ 1 & 3.979952 & -1.0878420 & 0.481080 \\ 1 & 3.183846 & -2.2963390 & -1.480904 \\ 1 & 2.642217 & -1.0782180 & -2.659325 \\ 1 & 1.460716 & -2.1317570 & -1.893633 \\ 1 & 1.906436 & 2.970858 & -1.1770300 \\ 1 & 0.205931 & 2.661378 & -1.5538050 \\ 1 & 0.644941 & 3.586516 & -0.0831310 \\ 1 & -1.966484 & 3.7363730 & -0.123127 \\ 1 & -3.408151 & 2.9157870 & -0.803313 \\ 1 & -3.025760 & 2.8227950 & 0.949525 \\ 6 & -2.165402 & -1.6462910 & 1.113792 \\ 6 & -2.271346 & -2.0858930 & -0.235825 \\ 6 & -1.031379 & -2.6544910 & -0.609191 \\ 6 & -0.852859 & -1.9363720 & 1.566771 \\ 6 & -0.148425 & -2.5490260 & 0.496624 \\ 1 & -2.941460 & -1.1517860 & 1.684788 \\ 1 & -3.135148 & -1.9649560 & -0.875314 \\ 1 & -0.789837 & -3.0551680 & -1.585999 \\ 1 & -0.447234 & -1.6963570 & 2.541871 \\ 1 & 0.885067 & -2.8679450 & 0.527854\end{array}$

$\begin{array}{lllr}\text { 15. } & & & \\ 22 & -0.952263 & 0.0963950 & 0.003341 \\ 6 & 3.378087 & -0.111429 & 1.1905270 \\ 6 & 4.082671 & -0.1268120 & -0.006894 \\ 6 & 3.369608 & -0.158911 & -1.1989340 \\ 6 & 1.972381 & -0.1635920 & -1.173986 \\ 7 & 1.288642 & -0.1350450 & 0.003334 \\ 6 & 1.205208 & -0.2080520 & -2.467614 \\ 6 & 1.980640 & -0.117060 & 1.1760580 \\ 6 & 1.223424 & -0.110716 & 2.4764140 \\ 7 & -1.239930 & 1.4730710 & 1.170063 \\ 6 & -1.287080 & 2.141137 & -0.0267720 \\ 6 & -1.392842 & 3.6482090 & -0.049688\end{array}$




$\begin{array}{llll}7 & -1.242872 & 1.4377550 & -1.203207 \\ 1 & 3.896284 & -0.0958040 & 2.143262 \\ 1 & 5.169186 & -0.1207580 & -0.010870 \\ 1 & 3.880805 & -0.1815810 & -2.155313 \\ 1 & 1.893251 & -0.2907870 & -3.313399 \\ 1 & 0.582074 & 0.683953 & -2.5881050 \\ 1 & 0.527743 & -1.0686270 & -2.489542 \\ 1 & 1.918690 & -0.1508780 & 3.319398 \\ 1 & 0.593411 & 0.780329 & 2.5629560 \\ 1 & 0.553865 & -0.9754080 & 2.541657 \\ 1 & -0.943069 & 4.0751290 & 0.851045 \\ 1 & -0.924242 & 4.0483060 & -0.953261 \\ 1 & -2.454629 & 3.9233910 & -0.065742 \\ 6 & -2.831851 & -1.1785230 & 0.776178 \\ 6 & -2.887122 & -1.1539450 & -0.649236 \\ 6 & -1.823859 & -1.9500520 & -1.141472 \\ 6 & -1.731790 & -1.9846840 & 1.154908 \\ 6 & -1.097511 & -2.4447530 & -0.029008 \\ 1 & -3.503740 & -0.6613680 & 1.448340 \\ 1 & -3.610206 & -0.6176740 & -1.249687 \\ 1 & -1.594175 & -2.1191260 & -2.186100 \\ 1 & -1.421211 & -2.1931560 & 2.171194 \\ 1 & -0.214901 & -3.0720180 & -0.073545\end{array}$

